\title{
Convex Partitions of Graphs induced by Paths of Order Three
}

\author{
C. C. Centenont \\ S. Dantas 非 \\ M. C. Dourado非 \\ D. Rautenbach \\ J. L. Szwarcfiter $\|^{1}$ \\ ${ }^{1}$ Instituto de Matemática, NCE, and COPPE, Universidade Federal do Rio de Janeiro, Rio de Janeiro, RJ, Brazil \\ ${ }^{2}$ Instituto de Matemática, Universidade Federal Fluminense, Niterói, RJ, Brazil \\ ${ }^{3}$ Institut für Optimierung und Operations Research, Universität Ulm, Ulm, Germany
}

received $9^{\text {th }}$ April 2010, revised $19^{\text {th }}$ October 2010, accepted 22 $2^{\text {nd }}$ November 2010.

A set $C$ of vertices of a graph $G$ is $P_{3}$-convex if $v \in C$ for every path $u v w$ in $G$ with $u, w \in C$. We prove that it is NP-complete to decide for a given graph $G$ and a given integer $p$ whether the vertex set of $G$ can be partitioned into $p$ non-empty disjoint $P_{3}$-convex sets. Furthermore, we study such partitions for a variety of graph classes.

Keywords: graph convexity; convex partition

\section{Introduction}

In [4] Bollobás considers "The spread of an infection on a square grid". Initially some squares of a square grid are infected. The infection spreads to an uninfected square if at least two of the neighbouring squares are infected. A natural combinatorial problem — solved using an elegant perimeter argument in [4] — is to determine the minimum number of initially infected squares such that eventually all squares become infected. This kind of spreading process has received considerable attention in a variety of contexts such as social influence [15], percolation [3], marketing strategies [10], and distributed computing [25]. In the present paper we study the above process under the perspective of graph convexity.

All graphs will be finite, simple, and undirected and we use common terminology and notation. Given a graph $G$ and a collection $\mathcal{C}$ of subsets of the vertex set $V(G)$ of $G$, the pair $(G, \mathcal{C})$ is a graph convexity if $\emptyset \in \mathcal{C}, V(G) \in \mathcal{C}$, and $\mathcal{C}$ is closed under intersection. The sets in $\mathcal{C}$ are called convex. The convex hull of some set $S$ of vertices of some graph $G$ with respect to some graph convexity $(G, \mathcal{C})$ is the unique smallest set $H_{\mathcal{C}}(S)$ in $\mathcal{C}$ containing $S$. Several natural graph convexities are defined in terms of paths. If $\mathcal{P}$ is a set of paths in some graph $G$ and $\mathcal{C}$ is the collection of all subsets $C$ of $V(G)$ such that, for every path $P$ in $\mathcal{P}$ between two vertices in $C$, the set $C$ contains all vertices of $P$, then $(G, \mathcal{C})$ is a graph convexity.

\footnotetext{
${ }^{\dagger}$ Email: carmenecos.ufrj.br.

‡Email: sdantas@im.uff.br.

§Email: mitreence.ufrj.br.

TEmail: dieter.rautenbach@uni-ulm.de.

"Email: jayme@nce.ufrj.br.

1365-8050 @ 2010 Discrete Mathematics and Theoretical Computer Science (DMTCS), Nancy, France
} 
Well-studied examples are geodetic convexity [18, 20], where $\mathcal{P}$ consists of all shortest paths, monophonic convexity [16, 19], where $\mathcal{P}$ consists of all induced paths, triangle-path convexity [8], and $m_{3}$-convexity [14]. In order to capture the above spreading process we choose $\mathcal{P}_{3}$ as the set of all paths of order 3 in some graph $G$. Specifically, we call a set $C$ of vertices in a graph $G P_{3}$-convex if, for every path uvw in $G$ with $u, w \in C$, the vertex $v$ belongs to $C$. (Note that all vertices of a path are distinct.) Equivalently, $C$ is $P_{3}$-convex in $G$ if and only if no vertex in $V(G) \backslash C$ has at least two neighbours in $C$. Let $\left(G, \mathcal{C}_{3}\right)$ denote the graph convexity where $\mathcal{C}_{3}$ is the collection of all $P_{3}$-convex sets of $G$. Convexities induced by paths of order 3 have been studied before in directed graphs and especially tournaments [17, 24, 26]. In [7] some results concerning $P_{3}$-convexity were obtained for undirected graphs.

There are some natural parameters associated with a graph convexity $(G, \mathcal{C})$. The convexity number of $(G, \mathcal{C})$ is the maximum cardinality of a proper convex subset of $V(G)$, and the hull number [6, 11, 13, 15, [18] of $(G, \mathcal{C})$ is the minimum cardinality of a set $S$ of vertices such that $H_{\mathcal{C}}(S)=V(G)$. Furthermore, if $(G, \mathcal{C})$ is defined in terms of a set $\mathcal{P}$ of paths in $G$ as explained above, then the geodetic number [9, 12] of $(G, \mathcal{C})$ is the minimum cardinality of a set $S$ of vertices such that the union of the vertex sets of all paths in $\mathcal{P}$ between vertices in $S$ equals $V(G)$. The geodetic number of the graph convexity $\left(G, \mathcal{C}_{3}\right)$ coincides with the well-studied 2-domination number [21,22]. The problem considered by Bollobás in [4] concerns the hull number of $\left(G, \mathcal{C}_{3}\right)$ where $G$ is the square grid $P_{n} \times P_{n}$. For further recent results concerning the hull number of $\left(G, \mathcal{C}_{3}\right)$, we refer the reader to [15, 6].

In the present paper we study the problem of partitioning the vertex set of a graph into $P_{3}$-convex sets. We establish the computational hardness of the corresponding decision problem in general and obtain results for several well-known graph classes [5]. Furthermore, we establish the hardness of the convexity number of $\left(G, \mathcal{C}_{3}\right)$. For analogous results concerning geodetic convexity, we refer to [2].

Before we proceed to the results, we recall some basic definitions. An independent set in a graph is a set of pairwise non-adjacent vertices. A clique in a graph is a set of pairwise adjacent vertices. A colouring of a graph is a partition of its vertex set into independent sets. The chromatic number of a graph $G$ is the minimum number $\chi(G)$ of independent sets into which its vertex set can be partitioned. A graph is 2 -connected if it is connected and the removal of a single vertex does not disconnect it. The $k$-th power of a graph $G$ is the graph $G^{k}$ with the same vertices as $G$ such that two distinct vertices are adjacent in $G^{k}$ if and only if their distance in $G$ is at most $k$. The union of two graphs $G_{1}$ and $G_{2}$ is the graph $G_{1} \cup G_{2}$ with vertex set $V\left(G_{1}\right) \cup V\left(G_{2}\right)$ and edge set $E\left(G_{1}\right) \cup E\left(G_{2}\right)$. For two graphs $G_{1}$ and $G_{2}$ with $V\left(G_{1}\right) \cap V\left(G_{2}\right)=\emptyset$, the join of $G_{1}$ and $G_{2}$ is the graph with vertex set $V\left(G_{1}\right) \cup V\left(G_{2}\right)$ and edge set $E\left(G_{1}\right) \cup E\left(G_{2}\right) \cup\left\{u v \mid u \in V\left(G_{1}\right) \wedge v \in V\left(G_{2}\right)\right\}$. A vertex $u$ in a graph $G$ is universal if it is adjacent to all vertices in $V(G) \backslash\{u\}$. A graph whose vertex set can be partitioned into an independent set and a clique is a split graph. A graph that does not contain an induced cycle of length at least 4 is chordal. A graph $G$ is an interval graph, if there is a collection of intervals of the real line indexed by the elements of $V(G)$ such that two distinct vertices are adjacent in $G$ if and only if the two corresponding intervals intersect. 


\section{Results}

A $P_{3}$-convex $p$-partition of a graph is a partition of its vertex set into $p$ disjoint non-empty $P_{3}$-convex sets. Clearly, the two trivial partitions into just one set containing all vertices of some graph $G$ and into $|V(G)|$ distinct sets each containing exactly one vertex from $V(G)$ are both $P_{3}$-convex partitions. Hence every graph $G$ has a $P_{3}$-convex $p$-partition for $p \in\{1,|V(G)|\}$. Not excluding these two trivial partitions it follows that - unlike as for many other graph partition notions such as for instance graph colourings there is no natural minimization or maximization problem related to the existence of $P_{3}$-convex partitions. In fact, the most natural problem in this context seems to be to characterize/determine those values of $p$ for which some given graph $G$ has a $P_{3}$-convex $p$-partition. This leads to the following decision problem.

\section{$P_{3}$-CONVEX PARTITION \\ Instance: $\quad$ A graph $G$ and a positive integer $p$. \\ Question: Does $G$ have a $P_{3}$-convex $p$-partition?}

Our first result is the hardness of this problem.

Theorem 2.1 $P_{3}$-CONVEX PARTITION is NP-complete even when restricted to split graphs.

Since we derive this result from Theorem 2.6 we postpone its proof for after that result.

As mentioned in the Introduction, the geodetic number and the hull number of the graph convexity $\left(G, \mathcal{C}_{3}\right)$ have already been studied in different contexts. In [7] it was proved that the following problem is NP-complete for general graphs.

\section{$P_{3}$-Convexity Number}

Instance: $\quad$ A graph $G$ and a positive integer $k$.

Question: $\quad$ Does $G$ have a $P_{3}$-convex set $C$ with $k \leq|C|<V(G)$ ?

It is not difficult to see that the construction given in [7] can be modified such that one obtains NPcompleteness already restricted to split graphs. For the sake of completeness, we give a direct proof.

Theorem 2.2 $P_{3}$-CONVEXITY NUMBER is NP-complete even when restricted to split graphs.

Proof: $P_{3}$-CONVEXITY NUMBER is clearly in NP. In order to complete the proof, we describe a reduction from 3-Dimensional Matching ([SP1] in [23]). An instance of 3-Dimensional Matching is a 3-uniform hypergraph $\mathcal{H}=(X, \mathcal{E})$ where $X$ has a partition $X_{1} \cup X_{2} \cup X_{3}$ such that every hyperedge in $\mathcal{E}$ contains exactly one vertex from each $X_{i}$ for $1 \leq i \leq 3$, and the question is whether $\mathcal{H}$ has a perfect matching, i.e. whether there is a set $\mathcal{M} \subseteq \mathcal{E}$ of hyperedges such that every vertex $u$ in $X$ is contained in exactly one of the hyperedges in $\mathcal{M}$.

Let $(X, \mathcal{E})$ be an instance of 3-Dimensional Matching. Clearly, we may assume that $|X| \equiv$ 0 mod 3 and that $|X|>3$. We construct a split graph $G$ and specify an integer $k$ such that the encoding length of $(G, k)$ is polynomially bounded in terms of the encoding length of $\mathcal{H}$ and $\mathcal{H}$ has a perfect matching if and only if $G$ has a $P_{3}$-convex set $C$ with $k \leq|C|<V(G)$. Let $G$ have vertex set $V(G)=$ $X \cup \mathcal{E}$ such that $X$ is a clique in $G, \mathcal{E}$ is an independent set in $G$, and $x \in X$ and $e \in \mathcal{E}$ are adjacent in $G$ if and only if $x \in e$. Let $k=\frac{|X|}{3}$.

If $\mathcal{M}$ is a perfect matching of $\mathcal{H}$, then $\mathcal{M}$ is a $P_{3}$-convex set in $G$ of order $k$. Conversely, let $U$ be a $P_{3}$-convex set in $G$ with $k \leq|U|<|V(G)|$. If $U$ contains two elements of $X$, then, by construction, 
$U=V(G)$, which is a contradiction. If $U$ contains a vertex in $X$ and a vertex in $\mathcal{E}$, then, by construction, $U$ contains two vertices in $X$, which is a contradiction. Since $k \geq 2$, this implies that $U \subseteq \mathcal{E}$. Since no vertex in $X$ is contained in two of the hyperedges in $U$, we have $|U| \leq \frac{|X|}{3}=k$. Hence $|U|=k$ and $U$ is a perfect matching of $\mathcal{H}$.

Our next result relates the existence of $P_{3}$-convex $p$-partitions of some graph to properties of suitable subgraphs. This result allows the design of efficient algorithms solving $P_{3}$-CONVEX PARTITION for classes of graphs, which have suitable decompositions. The statements (ii), (iii), and (iv) of Theorem 2.3 for instance can be used to efficiently determine the existence of $P_{3}$-convex $p$-partitions of cographs.

Theorem 2.3 Let $G$ be a graph of order $n$.

(i) If $H$ is a subgraph of $G, V_{1} \cup V_{2} \cup \cdots \cup V_{p}$ is a $P_{3}$-convex p-partition of $G, U_{i}=V_{i} \cap V(H) \neq \emptyset$ for $1 \leq i \leq p^{\prime}$, and $V_{i} \cap V(H)=\emptyset$ for $p^{\prime}+1 \leq i \leq p$, then $U_{1} \cup U_{2} \cup \cdots \cup U_{p^{\prime}}$ is a $P_{3}$-convex $p^{\prime}$-partition of $H$.

(ii) If $G$ is the disjoint union of the two graphs $G_{1}$ and $G_{2}$, then $G$ has a $P_{3}$-convex p-partition if and only if there is a $P_{3}$-convex $p_{1}$-partition of $G_{1}$ and a $P_{3}$-convex $p_{2}$-partition of $G_{2}$ such that $\max \left\{p_{1}, p_{2}\right\} \leq p \leq p_{1}+p_{2}$.

(iii) If $G$ arises by adding a universal vertex $u$ to some graph $G^{\prime}$, then $G$ has a $P_{3}$-convex p-partition if and only if $p=n$ or there is a set $U$ that is the union of the vertex sets of some components of $G^{\prime}$ such that $p=n-|U|$.

(iv) If $G$ is the join of two disjoint graphs $G_{1}$ and $G_{2}$ such that the orders of $G_{1}$ and $G_{2}$ are at least 2 , $G_{1}$ has $n_{1}$ isolated vertices, and $G_{2}$ has $n_{2}$ isolated vertices, then $G$ has a $P_{3}$-convex p-partition if and only if $p=1$ or $n-\min \left\{n_{1}, n_{2}\right\} \leq p \leq n$.

(v) If $G$ is the union of two graphs $G_{1}$ and $G_{2}$ and $\{u\}=V\left(G_{1}\right) \cap V\left(G_{2}\right)$, then $G$ has a $P_{3}$-convex p-partition if and only if there is a $P_{3}$-convex $p_{1}$-partition of $G_{1}$ in which exactly $p_{1}^{\prime}$ sets contain a neighbour of $u$ but not $u$ and a $P_{3}$-convex $p_{2}$-partition of $G_{2}$ in which exactly $p_{2}^{\prime}$ sets contain a neighbour of $u$ but not $u$ such that

$$
1+p_{1}^{\prime}+p_{2}^{\prime}+\max \left\{0, p_{1}-p_{1}^{\prime}-p_{2}^{\prime}-1, p_{2}-p_{1}^{\prime}-p_{2}^{\prime}-1\right\} \leq p \leq p_{1}+p_{2}-1 .
$$

Proof: (i) Since for every $1 \leq i \leq p^{\prime}$, no vertex $u \in V(G) \backslash V_{i}$ has two neighbours in $V_{i}$ and $U_{i} \subseteq V_{i}$, the partition $U_{1} \cup U_{2} \cup \cdots \cup U_{p^{\prime}}$ of $V(H)$ is a $P_{3}$-convex $p^{\prime}$-partition of $H$.

(ii) Let $\mathcal{P}: V_{1} \cup V_{2} \cup \cdots \cup V_{p}$ be a $P_{3}$-convex $p$-partition of $G$. Let $V_{1}^{1} \cup V_{2}^{1} \cup \cdots \cup V_{p_{1}}^{1}$ and $V_{1}^{2} \cup V_{2}^{2} \cup \cdots \cup V_{p_{2}}^{2}$ denote the two $P_{3}$-convex partitions of $G_{1}$ and $G_{2}$, respectively, that are induced by $\mathcal{P}$ exactly as in part (i). Clearly, $\max \left\{p_{1}, p_{2}\right\} \leq p \leq p_{1}+p_{2}$.

Conversely, let $V_{1}^{1} \cup V_{2}^{1} \cup \cdots \cup V_{p_{1}}^{1}$ and $V_{1}^{2} \cup V_{2}^{2} \cup \cdots \cup V_{p_{2}}^{2}$ be two $P_{3}$-convex partitions of $G_{1}$ and $G_{2}$, respectively. Let $p$ be such that $\max \left\{p_{1}, p_{2}\right\} \leq p \leq p_{1}+p_{2}$. Let

$$
V_{i}= \begin{cases}V_{i}^{1} \cup V_{i}^{2} & , 1 \leq i \leq\left(p_{1}+p_{2}\right)-p, \\ V_{i}^{1} & ,\left(p_{1}+p_{2}-p\right)+1 \leq i \leq p_{1}, \\ V_{\left(p_{2}-p\right)+i}^{2} & , p_{1}+1 \leq i \leq p .\end{cases}
$$


Since no vertex of $G$ has a neighbour in $V\left(G_{1}\right)$ and a neighbour in $V\left(G_{2}\right), V_{1} \cup V_{2} \cup \cdots \cup V_{p}$ is a $P_{3}$-convex $p$-partition of $G$.

(iii) Let $V_{1} \cup V_{2} \cup \cdots \cup V_{p}$ be a $P_{3}$-convex $p$-partition of $G$ with $p<n$. Let $u \in V_{1}$. Clearly, $\left|V_{i}\right|=1$ for $2 \leq i \leq p$. If $v w \in E\left(G^{\prime}\right)$ is such that $v \in V_{1}$, then $w \in V_{1}$. This implies that $U=V_{1} \backslash\{u\}$ is the union of the vertex sets of some components of $G^{\prime}$, and hence $p=n-|U|$.

Conversely, if $U$ is the union of the vertex sets of some components of $G^{\prime}$, then $U \cup\{u\}$ together with the $n-1-|U|$ sets each containing exactly one vertex from $V\left(G^{\prime}\right) \backslash U$ yields a $P_{3}$-convex $(n-|U|)$ partition of $G$.

(iv) Let $U$ be a proper subset of $V(G)$. It is easy to see that $U$ is $P_{3}$-convex if and only if $U$ does not contain two vertices from $V\left(G_{1}\right)$ or two vertices from $V\left(G_{2}\right)$, and does not contain a vertex $u_{1} \in V\left(G_{1}\right)$ and a vertex $u_{2} \in V\left(G_{2}\right)$ such that $u_{1}$ is not isolated in $G_{1}$ or $u_{2}$ is not isolated in $G_{2}$. Therefore the $P_{3}$-convex sets of $G$ are either of cardinality 1 or consist of one isolated vertex from $G_{1}$ and one isolated vertex from $G_{2}$, which easily implies the desired statement.

(v) Let $\mathcal{P}: V_{1} \cup V_{2} \cup \cdots \cup V_{p}$ be a $P_{3}$-convex $p$-partition of $G$. Let $\mathcal{P}_{1}: V_{1}^{1} \cup V_{2}^{1} \cup \cdots \cup V_{p_{1}}^{1}$ and $\mathcal{P}_{2}: V_{1}^{2} \cup V_{2}^{2} \cup \cdots \cup V_{p_{2}}^{2}$ denote the two $P_{3}$-convex partitions of $G_{1}$ and $G_{2}$, respectively, that are induced by $\mathcal{P}$ exactly as in (i). Since the partite set in $\mathcal{P}$ containing $u$ contributes a non-empty set to both induced partitions $\mathcal{P}_{1}$ and $\mathcal{P}_{2}$, we have $p \leq p_{1}+p_{2}-1$. Let $\mathcal{P}_{1}$ contain exactly $p_{1}^{\prime}$ sets that contain a neighbour of $u$ but not $u$, and let $\mathcal{P}_{2}$ contain exactly $p_{2}^{\prime}$ sets that contain a neighbour of $u$ but not $u$. If $V_{i}=V_{r}^{1} \cup V_{s}^{2}$ for some indices $i, r$, and $s$, then either (a) $u \in V_{r}^{1} \cap V_{s}^{2}$, or (b) $u \notin V_{r}^{1} \cup V_{s}^{2}$ and either $V_{r}^{1}$ or $V_{s}^{2}$ does not contain a neighbour of $u$.

Case (a) occurs exactly once. Case (b) occurs at least $p_{1}^{\prime}+p_{2}^{\prime}$ times for the partite sets in $\mathcal{P}_{1} \cup \mathcal{P}_{2}$ that contain a neighbour of $u$ but not $u$ and at least $\max \left\{0, p_{1}-p_{1}^{\prime}-p_{2}^{\prime}-1, p_{2}-p_{1}^{\prime}-p_{2}^{\prime}-1\right\}$ times for the remaining partite sets in $\mathcal{P}_{1} \cup \mathcal{P}_{2}$. Altogether, this implies that $p$ is at least $1+p_{1}^{\prime}+p_{2}^{\prime}+\max \left\{0, p_{1}-\right.$ $\left.p_{1}^{\prime}-p_{2}^{\prime}-1, p_{2}-p_{1}^{\prime}-p_{2}^{\prime}-1\right\}$.

Conversely, let $\mathcal{P}_{1}: V_{1}^{1} \cup V_{2}^{1} \cup \cdots \cup V_{p_{1}}^{1}$ and $\mathcal{P}_{2}: V_{1}^{2} \cup V_{2}^{2} \cup \cdots \cup V_{p_{2}}^{2}$ be two $P_{3}$-convex partitions of $G_{1}$ and $G_{2}$, respectively. We may assume that $u \in V_{1}^{1} \cap V_{1}^{2}$. Let $p_{1}^{\prime}$ and $p_{2}^{\prime}$ be as in the statement of $(\mathrm{v})$. Let $\mathcal{P}_{1}^{\prime}$ contain the partite sets in $\mathcal{P}_{1}$ that contain a neighbour of $u$ but not $u$ and let $\mathcal{P}_{2}^{\prime}$ contain the partite sets in $\mathcal{P}_{2}$ that contain a neighbour of $u$ but not $u$. Note that $p_{1}^{\prime}=\left|\mathcal{P}_{1}^{\prime}\right|$ and $p_{2}^{\prime}=\left|\mathcal{P}_{2}^{\prime}\right|$. Let $q=1+p_{1}^{\prime}+p_{2}^{\prime}+\max \left\{0, p_{1}-p_{1}^{\prime}-p_{2}^{\prime}-1, p_{2}-p_{1}^{\prime}-p_{2}^{\prime}-1\right\}$ and let $p$ be such that $q \leq p \leq p_{1}+p_{2}-1$. Let $V_{1} \cup V_{2} \cup \cdots \cup V_{q}$ be a partition of $V(G)$ such that one partite set is $V_{1}^{1} \cup V_{1}^{2}, p_{1}^{\prime}$ partite sets are the union of a partite set in $\mathcal{P}_{1}^{\prime}$ with one or zero partite set in $\mathcal{P}_{2} \backslash \mathcal{P}_{2}^{\prime}, p_{2}^{\prime}$ partite sets are the union of a partite set in $\mathcal{P}_{2}^{\prime}$ with one or zero partite set in $\mathcal{P}_{1} \backslash \mathcal{P}_{1}^{\prime}$, and $\max \left\{0, p_{1}-p_{1}^{\prime}-p_{2}^{\prime}-1, p_{2}-p_{1}^{\prime}-p_{2}^{\prime}-1\right\}$ partite sets are the union of one or zero partite set in $\mathcal{P}_{1} \backslash \mathcal{P}_{1}^{\prime}$ with one or zero partite set in $\mathcal{P}_{2} \backslash \mathcal{P}_{2}^{\prime}$. Such a partition clearly exists and is a $P_{3}$-convex $q$-partition of $G$. Now, replacing $p-q$ of the partite sets $V_{i}$ with $i \geq 2$ that are the union of two sets $V_{r}^{1}$ and $V_{s}^{2}$ with these two sets results in a $P_{3}$-convex $p$-partition of $G$. This completes the proof. 


\subsection{Powers of Cycles}

In this subsection, we consider $P_{3}$-convex partitions of the $k$-th power $C_{n}^{k}$ of the cycle $C_{n}$ of order $n$. Since the complete graph $K_{n}$ of order $n$ has a $P_{3}$-convex $p$-partition if and only if $p$ is 1 or $n$, we focus on non-complete cycle powers, i.e. $k<\left\lfloor\frac{n}{2}\right\rfloor$.

We denote the chromatic number of a graph $G$ by $\chi(G)$.

Theorem 2.4 Let $n, k \in \mathbb{N}$ be such that $n \geq 3$ and $1 \leq k<\left\lfloor\frac{n}{2}\right\rfloor$. $C_{n}^{k}$ has a $P_{3}$-convex p-partition if and only if

$$
\begin{aligned}
& \text { either } p=1, \\
& \text { or } k=1 \text { and } 3 \leq p \leq n, \\
& \text { or } k=1, n \geq 4, \text { and } p=2, \\
& \text { or } k \geq 2 \text { and } p \geq \chi\left(C_{n}^{2 k}\right) .
\end{aligned}
$$

Proof: Clearly, $C_{n}^{k}$ has the two trivial $P_{3}$-convex $p$-partitions for $p=1$ and $p=n$. If $k=1$, then every set of at most $n-2$ consecutive vertices of $C_{n}$ is $P_{3}$-convex in $C_{n}^{1}$. This clearly implies all statements concerning $k=1$. Now let $k \geq 2$. Let $U$ be a proper subset of $V\left(C_{n}^{k}\right)$ that is $P_{3}$-convex in $C_{n}^{k}$. If $U$ contains two consecutive vertices of $C_{n}$, then $U=V\left(C_{n}\right)$, which is a contradiction. If $U$ contains two distinct vertices $u$ and $v$ of distance at most $2 k$ in $C_{n}$, then $U$ contains all vertices on shortest paths in $C_{n}$ between $u$ and $v$ and hence $U=V\left(C_{n}\right)$, which is a contradiction. This implies that $U$ is an independent set of $C_{n}^{2 k}$. Conversely, every independent set $U$ of $C_{n}^{2 k}$ is a proper subset of $V\left(C_{n}^{k}\right)$ that is $P_{3}$-convex in $C_{n}^{k}$, because no vertex in $V\left(C_{n}\right) \backslash U$ has two neighbours in $U$. Therefore, $C_{n}^{k}$ has a $P_{3}$-convex $p$-partition if and only if $C_{n}^{2 k}$ has a $p$-colouring, which implies all statements concerning $k \geq 2$.

Theorem 2.4 refered to the chromatic number of $C_{n}^{2 k}$. We believe that the following result should be known. Since we have not been able to find a reference, we include the simple proof for completeness.

Lemma 2.5 If $n, k \in \mathbb{N}$ are such that $2 \leq k<\left\lfloor\frac{n}{2}\right\rfloor$, then $\chi\left(C_{n}^{k}\right)=\left\lceil\frac{n}{\left\lfloor\frac{n}{k+1}\right\rfloor}\right\rceil$.

Proof: A set of vertices $I$ that is independent in $C_{n}^{k}$ does not contain two vertices whose distance in $C_{n}$ is at most $k$. This implies that every independent set of $C_{n}^{k}$ contains at most $\left\lfloor\frac{n}{k+1}\right\rfloor$ vertices and hence $\chi\left(C_{n}^{k}\right)$ is at least the expression stated in the lemma. Let $n=(k+1) r+s$ for integers $r$ and $s$ with $0 \leq s \leq k$. We choose $r$ disjoint sets $V_{1}, V_{2}, \ldots, V_{r}$ each containing exactly $k+1$ consecutive vertices on $C_{n}$ such that each of the components of $C_{n}-\left(V_{1} \cup V_{2} \cup \cdots \cup V_{r}\right)$ contains either $\left\lfloor\frac{s}{r}\right\rfloor$ or $\left\lceil\frac{s}{r}\right\rceil$ many vertices. By construction, there is a colouring of $C_{n}^{k}$ using $k+1$ distinct colours for the vertices in $V_{1} \cup V_{2} \cup \cdots \cup V_{r}$ and $\left\lceil\frac{s}{r}\right\rceil$ distinct colours for the remaining vertices. This implies

$$
\chi\left(C_{n}^{k}\right) \leq(k+1)+\left\lceil\frac{s}{r}\right\rceil=\left\lceil(k+1)+\frac{s}{r}\right\rceil=\left\lceil\frac{(k+1) r+s}{r}\right\rceil=\left\lceil\frac{n}{r}\right\rceil=\left\lceil\frac{n}{\left\lfloor\frac{n}{k+1}\right\rceil}\right\rceil
$$

and hence $\chi\left(C_{n}^{k}\right)$ is at most the expression stated in the lemma, which completes the proof. 


\subsection{Split Graphs}

The next result captures the possibilities for the $P_{3}$-convex partitions of split graphs.

Theorem 2.6 Let $G$ be a split graph such that $V(G)$ is the disjoint union of an independent set $I$ and a clique K. Let $I_{\leq 1}=\left\{u \in I \mid d_{G}(u) \leq 1\right\}$ and let $I_{\geq 2}=I \backslash I_{\leq 1}$. Let $H=G^{2}\left[I_{\geq 2}\right]$.

$G$ has a $P_{3}$-convex $p$-partition if and only if $1 \leq p \leq 1+\left|I_{\leq 1}\right|$ or $\chi(H)+|K| \leq p \leq n$.

Proof: Let $\mathcal{P}: V_{1} \cup V_{2} \cup \cdots \cup V_{p}$ be a $P_{3}$-convex $p$-partition of $G$. Clearly, either $K$ is a subset of one partite set or every partite set contains at most one vertex from $K$. If $K$ is the subset of one partite set, say $V_{1}$, then $I_{\geq 2} \subseteq V_{1}$ and hence $1 \leq p \leq 1+\left|I_{\leq 1}\right|$. If every partite set contains at most one vertex from $K$, then no partite set contains a vertex from $I_{\geq 2}$ as well as a vertex from $K$. Furthermore, no partite set contains two vertices from $I_{\geq 2}$ at distance (at most) 2. Therefore, the partition of $I$ induced by $\mathcal{P}$ yields a colouring of $H$ and the partition of $K$ induced by $\mathcal{P}$ consists of $|K|$ sets. Altogether we obtain $\chi(H)+|K| \leq p \leq n$.

For the converse, let $p$ be such that $1 \leq p \leq 1+\left|I_{\leq 1}\right|$ or $\chi(H)+|K| \leq p \leq n$. If $1 \leq p \leq 1+\left|I_{\leq 1}\right|$, then $G$ has a $P_{3}$-convex $p$-partition in which one partite set consists of $I_{\geq 2}, K$, and a subset of $I_{\leq 1}$ of cardinality $1+\left|I_{\leq 1}\right|-p$, and all remaining $p-1$ partite sets consist of exactly one vertex from $I_{\leq 1}$. If $p=\chi(H)+|K|$, then $G$ has a $P_{3}$-convex $p$-partition, which, for every $u \in K$, contains one partite set that contains $\{u\} \cup\left(N_{G}(u) \cap I_{\leq 1}\right)$ together with some isolated vertices of $G$, and contains the partite sets of a partition of $I_{\geq 2}=V(H)$ into $\chi(H)$ sets that are independent in $H$. Clearly, replacing a partite set $V_{i}$ of this $P_{3}$-convex partition that satisfies $V_{i} \subseteq I_{\geq 2}$ with two non-empty subsets of $V_{i}$ results in a $P_{3}$-convex partition of $G$. Similarly, replacing a partite set $V_{i}$ of this $P_{3}$-convex partition that contains one vertex of $K$ and one vertex $u$ of $I_{\leq 1}$ with the two sets $V_{i} \backslash\{u\}$ and $\{u\}$ results in a $P_{3}$-convex partition of $G$. Iteratively applying these two operations, we obtain $P_{3}$-convex $p$-partitions for all $p$ with $\chi(H)+|K| \leq p \leq n$, which completes the proof.

We can now give the

Proof of Theorem 2.1: $P_{3}$-CONVEX Partition is clearly in NP. In order to complete the proof, we describe a reduction from Chromatic Number ([GT4] in [23]). Given an instance $(H, k)$ of ChroMATIC NUMBER, we construct a split graph $G$ and specify an integer $p$ such that the encoding length of $(G, p)$ is polynomially bounded in terms of the encoding length of $(H, k)$ and $H$ has a vertex colouring with $k$ colours if and only if $G$ has a $P_{3}$-convex $p$-partition. The vertex set of $G$ is the union of an independent set $I$ of order $|V(H)|$ and a clique $C$ of order $2|V(H)|+|E(H)|$. Let $f: V(H) \rightarrow I$ be bijective. For every vertex $u \in V(H)$, the vertex $f(u)$ has two neighbours $g(u)$ and $g^{\prime}(u)$ in $C$. Furthermore, for every edge $u v \in E(H)$, the vertices $f(u)$ and $f(v)$ have a common neighbour $g(u v)$ in $C$. In view of the order of $C$, we may assume that all specified vertices in $C$ are distinct, i.e. $C=\{g(u) \mid u \in V(G)\} \cup\left\{g^{\prime}(u) \mid u \in V(G)\right\} \cup\{g(u v) \mid u v \in E(G)\}$. Let $p=k+|C|$.

Now it follows easily from Theorem 2.6 that $H$ has a vertex colouring with $k$ colours if and only if $G$ has a $P_{3}$-convex $p$-partition, which completes the proof. 


\subsection{Chordal Graphs}

In this subsection, we study chordal graphs.

Theorem 2.7 Let $G$ be a 2-connected chordal graph.

(i) If $U$ is a proper subset of $V(G)$, then $U$ is a $P_{3}$-convex set of $G$ if and only if $U$ is an independent set of the square $G^{2}$ of $G$.

(ii) G has a $P_{3}$-convex $p$-partition if and only if $p=1$ or $p \geq \chi\left(G^{2}\right)$.

Proof: (i) Since the 'if'-part is obvious, we proceed to the proof of the 'only if'-part. For contradiction, we assume that $U$ contains two vertices $x$ and $y$ with distance at most 2 . We may assume that $U$ is the smallest $P_{3}$-convex set containing $x$ and $y$. Clearly, $U$ contains all vertices on shortest paths between $x$ and $y$ and $G[U]$ is connected. Since $U \neq V(G)$, there is an edge $u v \in E(G)$ with $u \in V(G) \backslash U$ and $v \in U$. Since $G$ is 2 -connected, there are two internally vertex-disjoint paths between $u$ and a vertex in $U \backslash\{v\}$. This implies the existence of a path $P: v_{1} v_{2} \ldots v_{l}$ in $G$ with $l \geq 3, v_{1}, v_{l} \in U$, and $v_{2}, \ldots, v_{l-1} \in V(G) \backslash U$. Choosing $P$ such that $l$ is minimum possible, the chordality of $G$ implies that $l=3$, which implies the contradiction $v_{2} \in U$.

(ii) By (i), $G$ has a $P_{3}$-convex $p$-partition if and only if $G^{2}$ has a $p$-colouring.

Note that if $G$ is a split graph and the set $I_{\leq 1}$ as defined in Theorem 2.6 is empty, then $G$ is a 2-connected chordal graph and the statement of Theorem 2.6 follows from Theorem 2.7 (ii).

By Theorem 2.7 $P_{3}$-convex partitions of a chordal graph $G$ are intimately linked to colourings of its square $G^{2}$. Unfortunately, it is even hard to approximate the chromatic number of squares of chordal

graphs of order $n$ within a factor of $O\left(n^{\frac{1}{2}-\epsilon}\right)$ for any $\epsilon>0$ [1]. Agnarsson et al. [1] establish such a hardness result already for squares of split graphs and their construction is quite similar to the construction used in the proof of our Theorem 2.1. Since interval graphs and squares of interval graphs are chordal, Theorem 2.7 allows to solve $P_{3}$-CONVEX PARTITION efficiently for 2-connected interval graphs.

Note that applying (v) of Theorem 2.3 to chordal graphs leads to some simplifications. In fact, if $G$ is a 2-connected chordal graph, $u \in V(G)$, and $V_{1} \cup V_{2} \cup \cdots \cup V_{p}$ is a $P_{3}$-convex $p$-partition of $G$ with $p \geq 2$, then, by Theorem 2.7, the partite set containing $u$ does not contain any neighbour of $u$ and every partite set contains at most one neighbour of $u$.

\section{Acknowledgements}

S. Dantas, M.C. Dourado, and J.L. Szwarcfiter acknowledge partial support by CNPq and FAPERJ, Brazilian research agencies. C.C. Centeno, M.C. Dourado, D. Rautenbach, and J.L. Szwarcfiter acknowledge partial support by the CAPES/DAAD Probral project "Cycles, Convexity, and Searching in Graphs". 


\section{References}

[1] G. Agnarsson, R. Greenlaw, and M.M. Halldórsson, On powers of chordal graphs and their colorings, Congr. Numerantium 144 (2000), 41-65.

[2] D. Artigas, M.C. Dourado, and J.L. Szwarcfiter, Convex partitions of graphs, Electronic Notes in Discrete Mathematics 29 (2007), 147-151.

[3] P. Balister, B. Bollobás, J.R. Johnson, and M. Walters, Random majority percolation, Random Struct. Algorithms 36 (2010), 315-340.

[4] B. Bollobás, The art of mathematics. Coffee time in Memphis, Cambridge University Press, 2006.

[5] A. Brandstädt, V.B. Le, and J.P. Spinrad, Graph classes: A survey, SIAM Monographs on Discrete Mathematics, 1999.

[6] C.C. Centeno, M.C. Dourado, L. Draque Penso, D. Rautenbach, and J.L. Szwarcfiter, Irreversible conversion of graphs, manuscript 2010.

[7] C.C. Centeno, M.C. Dourado, and J.L. Szwarcfiter, On the Convexity of Paths of Length Two in Undirected Graphs, Electronic Notes in Discrete Mathematics 32 (2009), 11-18.

[8] M. Changat and J. Mathew, On triangle path convexity in graphs, Discrete Math. 206 (1999), 91-95.

[9] G. Chartrand, E.M. Palmer, and P. Zhang, The geodetic number of a graph: A survey, Congr. Numerantium 156 (2002), 37-58.

[10] P. Domingos and M. Richardson, Mining the network value of customers, in Proceedings of the 7th ACM SIGKDD International Conference on Knowledge Discovery and Data Mining, 2001, 57-66.

[11] M.C. Dourado, J.G. Gimbel, J. Kratochvíl, F. Protti, and J.L. Szwarcfiter, On the computation of the hull number of a graph, Discrete Math. 309 (2009), 5668-5674.

[12] M.C. Dourado, F. Protti, D. Rautenbach, and J.L. Szwarcfiter, Some remarks on the geodetic number of a graph, Discrete Math. 310 (2010), 832-837.

[13] M.C. Dourado, F. Protti, D. Rautenbach, and J.L. Szwarcfiter, On the hull number of triangle-free graphs, SIAM J. Discrete Math. 23 (2010), 2163-2172.

[14] F.F. Dragan, F. Nicolai, and A. Brandstädt, Convexity and HHD-free graphs, SIAM J. Discrete Math. 12 (1999), 119-135

[15] P.A. Dreyer Jr. and F.S. Roberts, Irreversible $k$-threshold processes: Graph-theoretical threshold models of the spread of disease and of opinion, Discrete Appl. Math. 157 (2009), 1615-1627.

[16] P. Duchet, Convex sets in graphs. II: Minimal path convexity, J. Comb. Theory, Ser. B 44 (1988), 307-316.

[17] P. Erdős, E. Fried, A. Hajnal, and E.C. Milner, Some remarks on simple tournaments, Algebra Univers. 2 (1972), 238-245. 
[18] M.G. Everett and S.B. Seidman, The hull number of a graph, Discrete Math. 57 (1985), 217-223.

[19] M. Farber and R.E. Jamison, Convexity in graphs and hypergraphs, SIAM J. Algebraic Discrete Methods 7 (1986), 433-444.

[20] F. Harary and J. Nieminen, Convexity in graphs, J. Differential Geometry 16 (1981), 185-190.

[21] T.W. Haynes, S.T. Hedetniemi, and P.J. Slater, Fundamentals of domination in graphs, Marcel Dekker, 1998.

[22] J.F. Fink and M.S. Jacobson, $n$-domination in graphs, Graph Theory with Applications to Algorithms and Computer Science, John Wiley and Sons, 1985, 282-300.

[23] M.R. Garey and D.S. Johnson, Computers and intractability. A guide to the theory of NPcompleteness, W.H. Freeman and Company, 1979.

[24] D.B. Parker, R.F Westhoff, and M.J. Wolf, On two-path convexity in multipartite tournaments, Eur. J. Comb. 29 (2008), 641-651.

[25] D. Peleg, Local majorities, coalitions and monopolies in graphs: A review, Theor. Comput. Sci. 282 (2002), 231-257.

[26] J.C. Varlet, Convexity in tournaments, Bull. Soc. R. Sci. Liège 45 (1976), 570-586. 